Special Issue of the 6th International Congress \& Exhibition (APMAS2016), Maslak, Istanbul, Turkey, June 1-3, 2016

\title{
Investigation of Data Mining Processing Stage Effect on Performance in Interferometric Measurement System
}

\author{
V.G. BÖCEKÇI ${ }^{a * *}$ AND K. YILDIZ ${ }^{b}$ \\ ${ }^{a}$ Marmara University, Department of Electric and Electronics Engineering, Technology Faculty, \\ 34722 İstanbul, Turkey \\ ${ }^{b}$ Marmara University, Department of Computer Engineering, Technology Faculty, 34722 İstanbul, Turkey \\ Interferometric measuring systems are frequently used in determining the precise changes. The light of the \\ venture property is used in this system which is possible to perform measurements in the nanometer precision. \\ Analysis of data, which are taken from interferometric measurement systems, can be performed by fringe counting, \\ image processing. There are disadvantages of such kinds of methods which are cost, time and design challenge. \\ The most innovation in our study, the need of electronic circuit or image processing algorithm for determining \\ obtained values which are taken from measurement system as a result of processing part can be eliminated. \\ In processing step, arrangement of the data is quite important in terms of the achieved results. Through data \\ processing, it is provided to make faster analysis by the aid of increasing quantity of the data. The displacement \\ values which are taken by data processing show $90 \%$ success when comparing them with both electronic and image \\ processing techniques.
}

DOI: 10.12693/APhysPolA.131.46

PACS/topics: 07.05.Kf, 81.05.Bx, 06.30.Bp

\section{Introduction}

Interferometric measurement systems has features such as high accuracy, high resolution, and easy installation. These features are emerging as a significant advantage against other displacement measurement systems such as dilatometric and capacitive [1-3]. Displacement sign which is received from an interferometric system can be analyzed by various techniques [4]. Electronic counter circuit is used in the fringe counting technique, bright fringes in interferogram sign is detected by photoreceptor as resulting of excitation light. The number of electrical pulses are generated as a result of bright fringes at photoreceiver. The counting circuit is found and calculated the displacement amount [5]. The biggest disadvantage of the fringe counting method, it is affected too much from vibrations in environment. Another drawback is the generating incorrect photo signal because of the high level darkness in the bright-dark fringe transition. To eliminate this disadvantage often emerges as a complex electronic circuit design requirements [4]. Image processing technique can be used in displacement amount. In this technique, interferogram technique is saved as video signal. After pre filtering and thresholding, dark and bright fringes are obtained in binary level. The use of various filtering techniques are required in order to eliminate deterioration of the image signal. This technique has the disadvantage that the amount of video signal and analysis time [4]. The quality of the data which is used in data mining will affect the results.

Wide variety techniques are available that can be used

*corresponding author; e-mail: vgbocekci@marmara.edu.tr in data preprocessing. Data cleaning is used for removing the noise from data. Data conversion and data reduction is aimed to reduce the length of the data size with the help of removing or combined variables [6]. In database missing, inconsistent and noisy data can be found an important issue. This is called as a dirty data which is defined widely in literature. The data is converted to the suitable form in conversion phase. In general, the dimension reduction stage is used before the classification for feature extraction. In this work, the displacement data is imported to the Matlab as a binary formatted matrix. The raw displacement sign was analyzed as preprocessing stage. The number of binary ones which are formatted as raw in data matrix are grouped with developed algorithm. Each of the group was assigned as fringe found in the displacement. Besides this method is not requiring special design filter, it also demonstrates the superiority with giving much more faster results.

\section{Materials and method}

Interferogram signal containing information from the interferometric displacement measuring system was analyzed in Matlab platform. The matrix values which were obtained from interferogram sign, were defined by data preprocessing and significant values were grouped.

\subsection{Interferogram signal}

The interferogram signal with displacement information, the gray level values can be seen in Fig. 1.

The gray level values are converted to binary level with thresholding. Figure 2 shows the binary form of matrix.

The binary matrix was used to define the dark and bright fringe which is formed by zero and one values with the help of data preprocessing. 


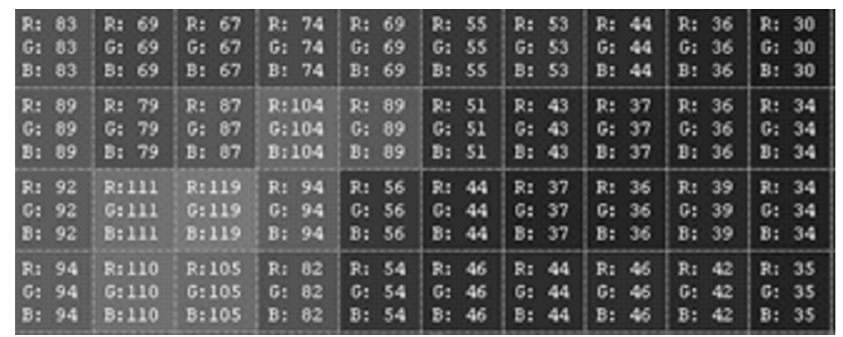

Fig. 1. Gray level matrix values section of interferogram signal.

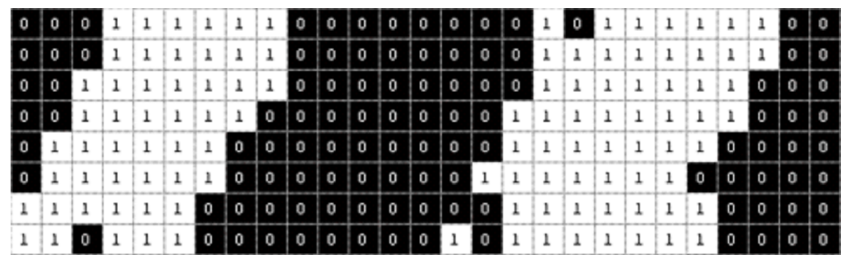

Fig. 2. Matrix values section of binary form.

\subsection{Data cleaning and reduction}

In the experimental stage of the data preprocessing, the data cleaning and reduction phase were applied to data. Also there should be conversion stage for different kinds of data. Because when the dimension increases, the data becomes noisy and irrelevant [7]. It is aimed to remove irrelevant, noisy, and faulty data from the matrix. The subgroups are found from the matrix that represents the " 1 " values. The designed system diagram is shown in Fig. 3. First, dimension reduction process was applied to remove the irrelevant points. Then the image data is represented as one dimension. The values from image matrix are converted to one dimension with zero and one values. In data matrix the starting and end values are found for " 1 " and " 0 " values. For the subarray, similar of the groups which are formed with " 1 " and cumulative total are found. The threshold level is defined for the cleaning noisy groups. The rest of the data is used to define fringe values.

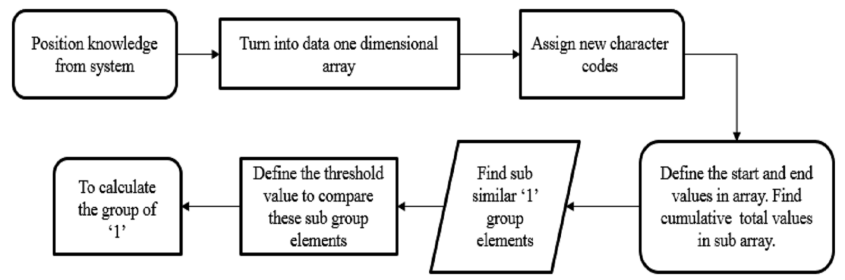

Fig. 3. The schematic diagram of data preprocessing stage and fringe counting.

\section{Results and discussion}

The number of group of the "1" was detected with the designed algorithm. This value was used for the calculation of the total displacement. The total displacement is dependent on the wavelength of the used source and defined with Eq. (1).

$$
\Delta L=\frac{\lambda}{2} N,
$$

where $\Delta L$ is the displacement; $N$ is number of ones, $\lambda$ is the laser wavelength. In Fig. 4a, it can be seen how to understand method. For this one the binary " 1 " is the noisy data which are not attended as a fringe so they are removed from the data because they are signed as noisy. Figure $4 \mathrm{~b}$ shows the group of " 1 " for fringes from the array matrix. The zero values are evaluated as noisy. So the whole matrix is evaluated by the count of the grouping number of "1". The noisy ones for both of zero and one are removed from the array to prevent the wrong identification.

(a) $100100000000000001000010000000000000000010100000000000000000001100000000 \ldots$ (b) $1111100111111111110111111111100011111111111111111111111111111111111 \ldots . . . . .$.

Fig. 4. The zero and one values: (a) binary "1" is the noisy data, (b) binary " 1 " shows fringes.

The relative error is about the $0.5 \%$ percentage with designed algorithm. In the experiment of the electronic fringe counting method, it is found as about $3 \%$. The data processing method gave more accurate results than the fringe counting. In video processing the relative error is about $0.3 \%$ however the designed method is simple and faster. Experimental results show that the data processing method is remarkable, it can be better with further improvements of the algorithm.

\section{Conclusion}

In this study, the data processing method is used to analyze the data which is obtained from interferometric system. The data processing method produce more sensitive results than electronic fringe counting method. The relative error is about the $3 \%$ in electronic fringe counting, however this value is decreased by $0.5 \%$ with data processing in measurement. Besides, when comparing the video processing and the data processing method, the relative error is small with video processing so the accuracy performance is high. But the designed algorithm comes toward simplicity and high data processing.

\section{References}

[1] N.I. Ismail, N.H. Ngajikin, N.F.M. Zaman, M.A.A.I. Azmi, N.N.A. Malik, N.M. Kassim, Telkomnika 12, 4 (2014).

[2] C. Lu, J.R. Troutman, T.L. Schmitz, J.D. Ellis, J.A. Tarbutton, in: Proc. 28th Annual American Society for Precision Engineering (ASPE) Conf., 2013, p. 364 .

[3] G. Bianchini, M. Barucci, T. Del Rosso, E. Pasca, G. Ventura, Precis. Eng. 44, 245 (2016).

[4] V. Bocekci, H.S. Varol, Acta Phys. Pol. A 127, 934 (2015).

[5] V. Bocekci, H.S. Varol, Acta Phys. Pol. A 121, 181 (2012).

[6] J. Han, M. Kamber, J. Pei, Data Mining Concept and Techniques, 3rd ed., Morgan Kaufmann, 2011.

[7] K. Yıldız, Y. Camurcu, B. Dogan, Akademik Bilişim, 10-12 February 2010, Muğla University, Muğla 2010. 